\title{
Letter to the Editor regarding the article "Meta-analysis of efficacy and safety of continuous saline bladder irrigation compared with intravesical chemotherapy after transurethral resection of bladder tumors"
}

\author{
Mohammad Hossein Panahi ${ }^{1}$ (D)
}

Received: 11 January 2019 / Accepted: 13 January 2019 / Published online: 1 February 2019

○) Springer-Verlag GmbH Germany, part of Springer Nature 2019

Dear Editor,

I recently reviewed the article by Zhou et al. [1] published in the January 2019 issue of the World Journal of Urology, entitled "Meta-analysis of efficacy and safety of continuous saline bladder irrigation compared with intravesical chemotherapy after transurethral resection of bladder tumors". I assessed its methodological quality using 16-item AMSTAR2 [2] appraisal tool. According to AMSTAR2, the study scored 11 items out of 16 while lost points from items 2, 9, 12, 13 and 14 which were related to the issues of protocol registry, risk of bias (ROB) and heterogeneity, respectively. ROB was well assessed by appropriate tools but its result was not accounted into the analysis through meta-regression/subgroup analysis or sensitivity analysis which may due to the paucity of the included studies. No commentary on the likely impact of ROB in discussion as well. In addition, although no heterogeneity was observed, it was not mentioned in the discussion part.

I suppose there was a typographical error to report "OR of -1.01 and $95 \%$ CI of -2.96 to $0.94(p=0.31)$ " for the median period to the first recurrence while it was mean difference not odds ratio.

Finally, to evaluate clinical significance, prediction interval (PI) was proposed in contrast to statistical significance presented by confidence interval (CI). I suggest that authors calculate prediction interval for evaluating clinical significances [3] for macrohematuria, frequency of urination and bladder irritation symptoms which were considered statistically significant.

As a conclusion, this was a well-written paper and most items were followed appropriately; non-critical weaknesses were observed there, so based on AMSTAR2, this study is classified as "Moderate" quality.

\section{Compliance with ethical standards}

Conflict of interest The authors declare that they have no conflicts of interests.

\section{References}

1. Zhou Z, Zhao S, Lu Y, Wu J, Li Y, Gao Z, Yang D, Cui Y (2019) Meta-analysis of efficacy and safety of continuous saline bladder irrigation compared with intravesical chemotherapy after transurethral resection of bladder tumors. World J Urol. https://doi. org/10.1007/s00345-019-02628-7

2. Shea BJ et al (2017) AMSTAR 2: a critical appraisal tool for systematic reviews that include randomised or non-randomised studies of healthcare interventions, or both. BMJ 358:j4008

3. Borenstein $\mathrm{M}$ et al (2011) Introduction to meta-analysis. Wiley, Hoboken

Publisher's Note Springer Nature remains neutral with regard to jurisdictional claims in published maps and institutional affiliations.

Mohammad Hossein Panahi

mohammadpanahi55@yahoo.com

1 Department of Epidemiology and Biostatistics, School of Public Health, Tehran University of Medical Sciences, Tehran, Iran 